\title{
Neutral sphingomyelinase (SMPD3) deficiency disrupts the Golgi secretory pathway and causes growth inhibition
}

\author{
Wilhelm Stoffel ${ }^{\star, 1,2}$, Ina Hammels ${ }^{1,2}$, Bitta Jenke ${ }^{1}$, Erika Binczek², Inga Schmidt-Soltau ${ }^{1}$, Susanne Brodesser ${ }^{2}$, Astrid Schauss ${ }^{2}$, \\ Julia Etich ${ }^{3}$, Juliane Heilig ${ }^{3}$ and Frank Zaucke ${ }^{3,4}$
}

Systemic loss of neutral sphingomyelinase (SMPD3) in mice leads to a novel form of systemic, juvenile hypoplasia (dwarfism). SMPD3 deficiency in mainly two growth regulating cell types contributes to the phenotype, in chondrocytes of skeletal growth zones to skeletal malformation and chondrodysplasia, and in hypothalamic neurosecretory neurons to systemic hypothalamuspituitary-somatotropic hypoplasia. The unbiased smpd3-/- mouse mutant and derived smpd3-/- primary chondrocytes were instrumental in defining the enigmatic role underlying the systemic and cell autonomous role of SMPD3 in the Golgi compartment. Here we describe the unprecedented role of SMPD3. SMPD3 deficiency disrupts homeostasis of sphingomyelin (SM), ceramide (Cer) and diacylglycerol (DAG) in the Golgi SMPD3-SMS1 (SM-synthase1) cycle. Cer and DAG, two fusogenic intermediates, modify the membrane lipid bilayer for the initiation of vesicle formation and transport. Dysproteostasis, unfolded protein response, endoplasmic reticulum stress and apoptosis perturb the Golgi secretory pathway in the smpd3-/- mouse. Secretion of extracellular matrix proteins is arrested in chondrocytes and causes skeletal malformation and chondrodysplasia. Similarly, retarded secretion of proteo-hormones in hypothalamic neurosecretory neurons leads to hypothalamus induced combined pituitary hormone deficiency. SMPD3 in the regulation of the protein vesicular secretory pathway may become a diagnostic target in the etiology of unknown forms of juvenile growth and developmental inhibition.

Cell Death and Disease (2016) 7, e2488; doi:10.1038/cddis.2016.385; published online 24 November 2016

Phospholipids (PLS), sphingolipids (SLS) and cholesterol form the complex architecture of mammalian membrane lipid bilayers. In addition, PLs and SLs are substrates of membraneassociated phospholipases and phosphodiesterases, the reaction products of which act as lipid second messengers. Acid sphingomyelinase (aSMase, SMPD1) and neutral SMases (nSMases, sphingomyelin (SM) phosphodiesterases, SMPD2-5) ${ }^{1-4}$ hydrolyze SM to phosphocholine and Cer. Cers are regarded lipid second messengers in rather divergent pathways of cellular signaling in growth and development, including triggering tumor-suppressive and anti-proliferative cellular processes. ${ }^{5,6}$ However, this tenet has been challenged ${ }^{7-13}$ and Cer functions have remained enigmatic.

The null-allelic smpd1- (Niemann-Pick, type A), ${ }^{14,15}$ smpd2 ${ }^{11}$ and smpd $3^{13}$ mouse-mutants have served as unbiased genetic tools in studies on the systemic role of mammalian SMPDs in cellular SM metabolism and its pathophysiology. The current study elaborated the pivotal role of SMPD3 utilizing the smpd3-/- mouse, which is characterized by the retardation of systemic and skeletal growth and development. In wild-type mice, the smpd3 mRNA is ubiquitously expressed. ${ }^{2,16,}$ The absence of SMPD3 in hypothalamic secretory neurons inhibited the secretion of proteo-hormones, slowed down the hypothalamus-pituitary-growth axis, and triggered systemic growth retardation resulting in a novel juvenile dwarf phenotype. ${ }^{13}$ The autonomous expression of SMPD3 in chondrocytes was shown by functional reconstitution of SMPD3 in smpd3-/- chondrocytes, expressing smpd3 as transgene, driven by the chondrocyte-specific Col2a1 promoter in the smpd3-/- mutant. $^{16}$

Here, we describe a novel molecular mechanism underlying the bifurcated systemic and cell autonomous SMPD3 deficiency. We first documented the dominant role of SMPD3 and defined the subcellular localization in the Golgi compartment (GC), imperative for unraveling the molecular role of SMPD3 in mammalian cells. Our finding is ad variance with the proposed plasma membrane (PLM) topology of SMPD3. ${ }^{18,19}$

We next focused our study on the cell-specific growth regulation of SMPD3 in primary chondrocytes of skeletal growth plates of p 16 control and smpd3 - / - mice, corresponding to approximately age 4 years in human development. ${ }^{20,21}$ Chondrocytes are competent secretory cells during the growth phase with an abundant secretion of extracellular matrix proteins (ECMs) for enchondral ossification in longitudinal growth. ${ }^{20}$ Chondrocytes in culture have proven most powerful in exploring molecular features of growth and development. ${ }^{22}$

We discovered the pivotal role of SMPD3 in Golgi vesicular protein transport. Inactivation of smpd3 stalled Golgi protein

\footnotetext{
${ }^{1}$ Center of Molecular Medicine (CMMC), Laboratory of Molecular Neurosciences, Center for Biochemistry, Faculty of Medicine, University of Cologne, Cologne, Germany; ${ }^{2}$ Cluster of Excellence, Cellular Stress Response in Aging-Related Diseases (CECAD), University of Cologne, Cologne, Germany; ${ }^{3}$ Department of Pediatrics and Adolescent Medicine, Experimental Neonatology, Center for Biochemistry, Faculty of Medicine, University of Cologne, Cologne, Germany and ${ }^{4}$ Dr Rolf M Schwiete Research Unit for Osteoarthritis, Orthopedic University Hospital, Friedrichsheim gGmbh, Frankfurt/Main, Germany

${ }^{*}$ Corresponding author: W Stoffelr, Center of Molecular Medicine (CMMC), Laboratory of Molecular Neurosciences, Center for Biochemistry, Faculty of Medicine, University of Cologne, Joseph-Stelzmann-Str. 52, Cologne D-50931, Germany. Tel: +49 221478 6881; Fax: +49 221478 6882; E-mail: wilhelm.stoffel@uni-koeln.de Received 19.8.16; revised 21.10.16; accepted 24.10.16; Edited by R De Maria
} 
transport, disrupted proteostasis, induced ER stress and compromised chondrocyte function, leading to apoptosis and ultimately to skeletal malformation and severe chondrodysplasia.

We determined the lipidome of primary chondrocytes of control and smpd3-/- mice in the growth phase as the structural platform for the functional analysis of SMPD3 in the Golgi secretory pathway (GSP). Our studies suggest a concerted action of SMPD3 and SMS1 in the Golgi complex, which maintains SM/phosphatidylcholine and ceramide (Cer) diacylglycerol (DAG) homeostasis during remodeling of the Golgi membrane lipid bilayer for vesicular transport. This homeostasis is perturbed in SMPD3-deficient GC.

Our studies delineate a novel function of SMPD3 in the lipiddriven formation of vesicle carriers in the GSP during growth and development, and provide insight into the molecular pathology of SMPD3 deficiency leading to an unprecedented mechanism of growth inhibition and to retarded development, manifested juvenile dwarfism and osteochondrodysplasia.

\section{Results}

SMPD3 is the key neutral sphingomyelinase, localized in detergent-insoluble membrane domains of the Golgi complex. We first ascertained the subcellular topology, a prerequisite for exploring the mechanistic role of SMPD3. Biochemical and immunohistochemical analyses conclusively proved the absence of SMPD3 in smpd3-/chondrocytes (Figures 1a and d). SMPD3 topology was restricted to the Golgi complex in different control cell types: in primary control chondrocytes (Figure 1c), peritoneal macrophages (Figure 1e) and C57BI/6 EMFIs (Figure 1f). Colocalization with SMS1 and Golgi-specific marker K58 ascertained the allocation to the GC (Figures $1 \mathrm{~g}$ and $\mathrm{h}$ ). SMPD3 and SMS1 resided in the GC of control chondrocytes and SMPD3 overexpressing HEK293 cells (Figures 1i and j).

To further substantiate the Golgi topology of SMPD3 and of SMS1, we used mouse brain, which shows highest SMPD3 expression of all tissues. ${ }^{2,16}$ The Golgi fraction of the premyelinating brain of (p14) control and smpd3-/- mice was further separated into detergent (Triton X-100)-insoluble membrane domains (DIMs) and subnatant for WB (Figures 1k-n). More than two-thirds of total SMPD3 resided in DIMS of control GC (Figure 1k), SMPD3 was absent in smpd3-/- GC (Figure 1m). Similarly, two-thirds of total SMS1 was concentrated in DIMs of control GC (Figure 11), but was equally distributed in SMPD3deficient DIMs and subnatant (Figure 1n).

To explore whether SMPD3 is required selectively for the canonical Golgi-mediated or non-canonical secretory pathway, we analyzed the secretion of several cytokines into the medium of control and smpd3-/- peritoneal macrophages, unstimulated (Figure 10) and stimulated (Figure 1p) with lipopolysaccharide (LPS). The unchanged secretion of cytokines in SMPD3-deficient macrophages clearly indicated SMPD3 function to be restricted to the canonical GSP.

A valuable tool in dissecting Golgi localization, morphology and vesicle formation is exposure of chondrocytes to Brefeldin (BFA). We followed the time-resolved disintegration by IHC of the GC of SMPD3 and K58 (Supplementary Figure S2a), and
Col2a and cartilage oligomeric matrix protein (COMP), in control and smpd3-/- chondrocytes (Supplementary Figure S2b). Golgi membrane stacks disintegrated, vesiculated and fused with ER membranes within 5-30 min.

We then assessed the contribution of bona fide SMPD4 (ref. 3) to cellular nSMase activity in HEK293 cell clones, stably transfected with full-length smpd4-egfp, with threefold to an eightfold overexpression, documented by qRT-PCR (Supplementary Figure S1a). SMPD4-EGFP colocalized largely with K58 in the GC (Supplementary Figure S1c). Surprisingly, the sensitive radioactive nSmase assay revealed an unchanged basal nSMase activity in the postnuclear fraction of all smpd4-egfp overexpressing cell clones (Supplementary Figure S1b). Our biochemical experiments and IHC were unable to substantiate nSMase activity of SMPD4.

The SMPD3-SMS1 cycle regulates SM homeostasis and the Cer- and PLC-independent DAG pool in the GC. Current techniques preclude time and space resolution between the transfers of de novo synthesized Cer from the ER into the GC, and Cer, released locally by SMPD3 hydrolysis from SM of Golgi membrane domains. To get insight into the metabolic interrelationship of SM/Cer and PC/DAG, we focused on their analysis in control and smpd3-/- primary chondrocytes in culture. We next analyzed the PL-classes of control and smpd3-/- chondrocytes, which were separated by highperformance thin layer chromatography (HPTLC).

Basically, Cer and DAG concentrations in the lipidome are very low. In smpd3-/- chondrocytes, the molar ratios of SM and Cer were reduced to one-half and one-third, respectively (Figures 2i-k).

Mass spectrometry, using selective ion monitoring (SIM), was applied for identification and quantification of HPTLCseparated Cer bands (m/z 264 and m/z 266), respectively. In $\mathrm{GC}$, the pattern of fatty acid substituents of $\mathrm{DH}$-Cer of control and smpd3-/- chondrocytes represents de novo synthesized $\mathrm{DH}-\mathrm{Cer}$, which is markedly different from that of Cer. $\mathrm{DH}-\mathrm{Cer}$ in control and smpd3-/- chondrocytes contained only saturated 16:0-22:0 acyl-groups, Cer species predominantly very long chain 24:0, 24:4 and 26:1-acyl-residues as substituents (Figures $2 \mathrm{~b}$ and $\mathrm{c}$ ).

$\mathrm{PC}$, the donor in the SMS1 catalyzed transfer of the phosphoryl-choline head group for reconstitution of SM, in Golgi of control and smpd3 -/- chondrocytes lacked polyunsaturated fatty acid substituted DAGs, which are closely similar to species in the pool of free DAGs. Sn1-18:0-sn2-20:4DAG, released by PLC $\gamma$-specific hydrolysis of PLM-bound PIP2 (Supplementary Figure S3f) was hardly detectable in the DAG pool of the Golgi complex (Figure 2e). The hydrophobic DAG core of PS, PI and PE in the Golgi membrane bilayer of chondrocytes of p16 control and smpd3-/- mice remained unchanged (Figures 2f-h).

SMPD3 deficiency disrupts the GSP. Primary chondrocytes of control and smpd3-/- mice (p16) in culture show an abundant ECM protein synthesis, macro-vesicular transport and secretion. We followed Golgi vesicular transport and secretion of major ECM proteins, Col2a, the main fibrillar collagen species, perifibrillar COMP, Matrilin (Matn) 3, CollX and ColVI in primary chondrocytes of p16 control and 
a

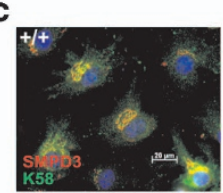

b

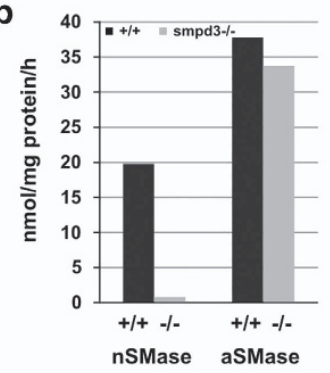

$e_{+/+}$
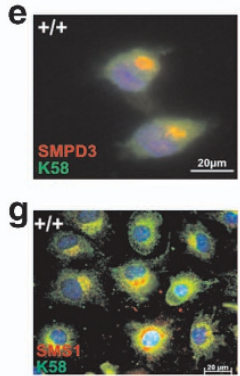

d
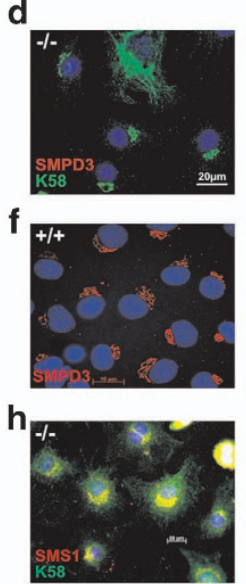

i
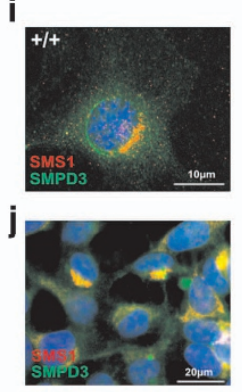
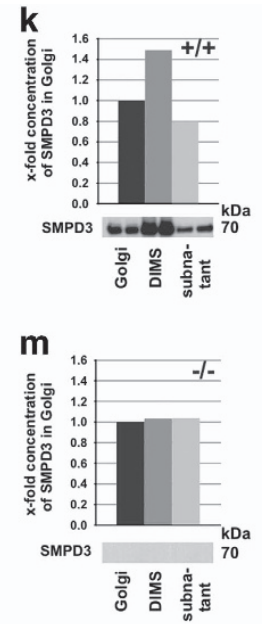

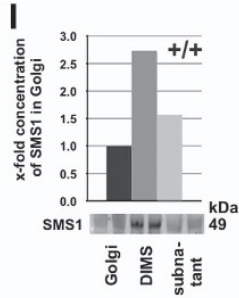

n

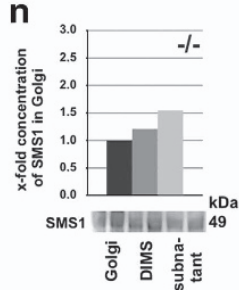

o

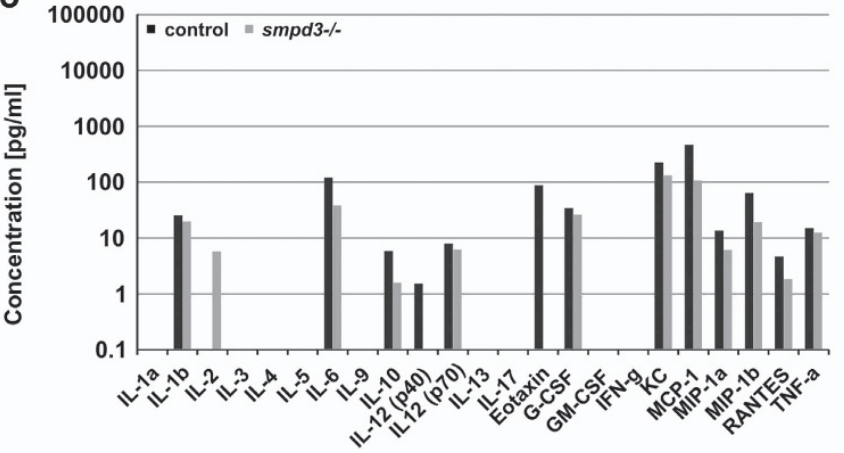

p

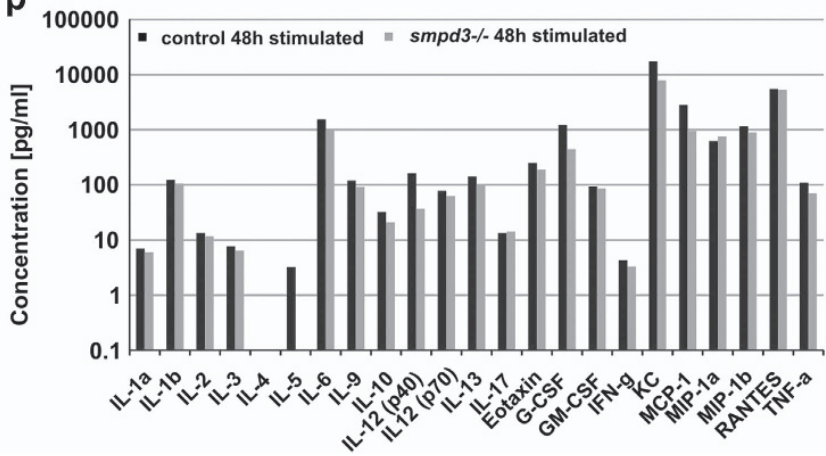

Figure 1 SMPD3 is the key nSMase segregated into DIMS of the GC. (a) Absence of SMPD3 in WB of lysates of smpd3 - / - chondrocytes. (b) Quantitative assessment of nSMase and aSMase activity in control and smpd3 $-/-$ chondrocytes using radioactive enzyme assays, $N=10$. Fluorescence images demonstrate colocalization of SMPD3 (red) and K58 (green) (c) in control chondrocytes (d), absence of SMPD3 in smpd3-/- chondrocytes. (e) Colocalization of SMPD3 (red) and K58 (green) in peritoneal macrophages and (f) Golgi localization of SMPD3 in C57BL/6 embryonal fibroblasts, ( $\mathbf{g}$ and $\mathbf{~}$ ) colocalization of SMS1 (red) and K58 (green) in control and smpd3 - /chondrocytes, $N=4$. Colocalization of SMPD3 (green) and SMS1 (red) in (i) control chondrocytes and (j) smpd3 transfected HEK293 cells, $N=3$. (k-n) Representative densitometric evaluation of WB signals of SMPD3 and SMS1 in total Golgi-, DIM- and subnatant fractions of (k and I) control and ( $\mathbf{m}$ and $\mathbf{n}) \mathrm{smpd} 3-/-\mathrm{p} 14$ brain. In all, $100 \mu \mathrm{g}$ aliquots of total protein were applied to each lane. Signals were evaluated by densitometry and normalized to SMPD3 and SMS1 present in the Golgi fraction, $N=4$. The noncanonical secretory pathway was assayed within a multi-cytokine assay quantifying secretion of cytokines into the growth-medium of peritoneal macrophages, (o) unstimulated and (p) stimulated with LPS

smpd3-/- mice by IHC (Figures 3a-h). Control chondrocytes effectively secreted Col2a, Matn3, CoIVI and CollX and formed a high-density ECM network. Col2a secretion was stalled in smpd3-/- chondrocytes and the intercellular fibrillar network nearly absent. COMP was distributed throughout the intracellular space of control chondrocytes. Arf and $\beta$-Cop1, markers of Golgi small vesicular transport carriers, displayed indistinguishable Golgi localization in control and smpd3-/- chondrocytes (Figure 3e).

$\mathrm{IHC}$ of the other dominant growth regulating cell type, hypothalamic neurosecretory neurons in arcuate $N$. and periventricular $N$., using antibodies against growth hormonereleasing hormone $(\mathrm{GHRH})$ and melanocyte-stimulating hormone $(\mathrm{MSH})$, revealed smpd3-/- neurons heavily loaded with proteo-hormones, but low abundance in control neurons (Figures $3 f$ and g), which confirms previous immunohistochemical results. ${ }^{13}$

Inhibited protein transport, dysproteostasis, ER stress and apoptosis in SMPD3-deficient chondrocytes. We next studied the cellular response to the stalled protein transport along the GSP. ER stress is measured by activation of unfolded protein response (UPR) and visualized by the accumulation of misfolded proteins in the lumen of the tubular ER system directly by transmission electron microscopy (EM) (for review Oslowski and Urano ${ }^{24,25}$ and Riggs et al. ${ }^{26}$ ).

EM of p16 control chondrocytes revealed normal rough endoplasmic reticulum (rER) tubular network (Figures 4a-c), but dilated and giant ER cisternae in smpd3-/- chondrocytes, the cytoplasm filled with macro-vesicular structures, displacing and disrupting the rER (Figures 4d-f). Enhanced expression of ER stress sensor/transducer activating transcription factor 6 (ATF6), following stress-induced proteolysis indicated activation of UPR in smpd3-/- chondrocytes, but UPR responder protein pIRE remained unchanged in WB (Figures $4 \mathrm{~g}$ and $\mathrm{h}$ ). Annexin $\mathrm{V}$ staining of smpd3-/chondrocytes (Figure 4j), FACS analysis of single-cell control and smpd3-/- p16 chondrocytes in culture (Figures 4k-m) and the terminal deoxynucleotidyl transferase dUTP nick end labeling (TUNEL) assay ${ }^{27}$ in sections of long bone epiphyses of smpd3-/- mice (Figures $4 n-s$ ), monitored onset of apoptosis, which resulted in severe osteo-chondrodysplastic 
4
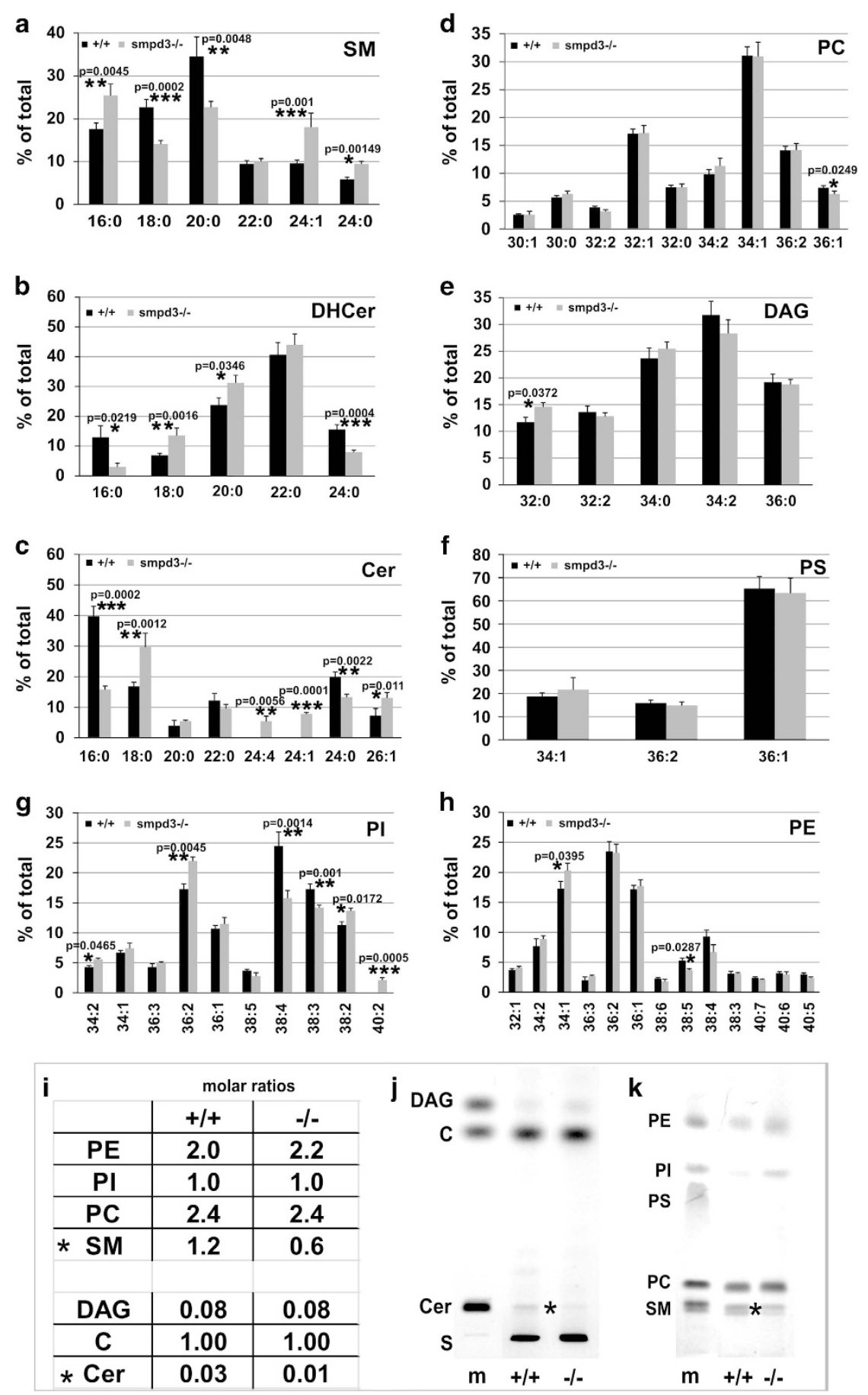

Figure 2 Molar ratios of SM and Cer are reduced in Golgi-lipidome of smpd3 - / - chondrocytes. MS/MS profiling of (a-h) species of SM (a), dihydroceramide (DH-Cer) (b), Cer (c), PC (d), DAG (e), phosphatidylserine (PS) (f), PI (g) and phosphatidylethanolamine (PE) (h) of Golgi fraction of control and smpd3 - / - chondrocytes. (i) Molar ratio of main PL classes (PC, PI and PE) and SM, Cer, C and DAG. (j and $\mathbf{k}$ ) Thin layer chromatographic separation of lipid extract of control and smpd3-/- chondrocytes. Solvent system: cyclohexane/ethylacetate $3: 2 \mathrm{v} / \mathrm{v}$ (j); two runs in chloroform/ethanol/water/triethylamine 30:35:7:35 v/v/v (k). $N=3$

malformation, paradigmatically documented in sections of decalcified femural epiphysis of smpd3-/- mice (Figure $4 \mathrm{u}$ ).

Smpd3-/- gene expression in primary chondrocytes. Next, we studied steady-state gene expression in chondrocytes of p16 control and smpd3-/- littermates by real-time PCR of (a) key enzymes of SM metabolism (Figure 5a), of ceramide synthases (cerS) and fatty acid elongases (elovl) (Figure 5b), (b) growth and transcription factors regulating chondrocyte differentiation, (c) ECM proteins and (d) sec23, essential for protein transport (Figure 5c). Expression of comp, bone morphogenetic protein 1 (bmp1), a procollagenase, and growth factor $b m p 4$, required for cartilage formation was downregulated (Figure 5c). 

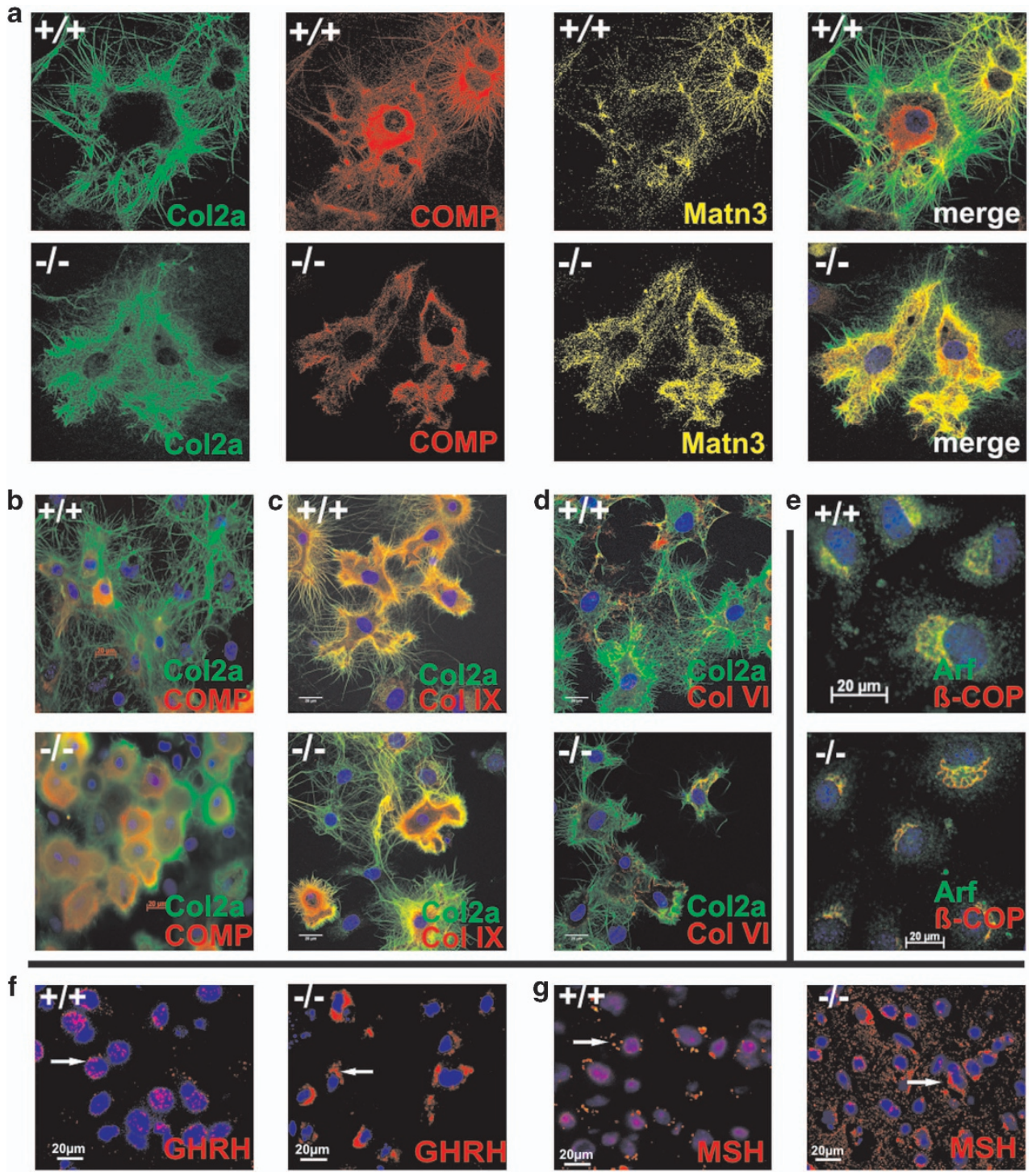

Figure 3 SMPD3-deficient primary chondrocytes display inhibited secretion of ECM proteins. Fluorescence images of p16 control and smpd3 - / - primary chondrocytes, using following antibodies for triple staining: (a) Col2a (green), COMP (red), and Matn3 (yellow), and double staining: merged images of (b) Col2a (green), COMP (red), (c) Col2a (green), CollX (red), (d) Col2a (green), ColVI (red), and (e) Arf (green), $\beta$-Cop1 (red) double stained primary chondrocytes in culture. Inhibited GSP in hypothalamic secretory neurons of control and smpd3 - / - mice: fluorescence images of (f) GHRH secreting neurons in control, storage of GHRH in smpd3 - / - neurons using anti-GHRH, (g) MSH in control and storage of MSH in proopiomelanocortin (POMC) expressing smpd3-/- neurons. $N=3$

Complementary WB analysis of structural proteins Col2a, COMP, Golgi-transport regulatory proteins $\beta$-Cop1 and Arf, Igf-1, IgfR-1 and SMS1 (Figures 5d-j). SMS1 expression was increased nearly threefold (Figure 5j), matching the increased SMS1 enzyme activity in the lysate of smpd3-/- chondrocytes (Figure 5k).

Equal amounts of lipid total extracts of enzyme assays were separated by HPTLC and visualized by charring lipid bands (Figure 5l), different from enzyme assay of kidney, which shows equal amounts of de novo synthesized fluorescent SM (Figures $5 \mathrm{~m}$ and $\mathrm{n}$ ).

\section{Discussion}

This study is focused on the mechanism underlying SMPD3 deficiency, which causes systemic and cell-specific growth inhibition, a novel form of juvenile dwarfism. We used the unbiased smpd3-/- mouse model. In this study, primary chondrocytes in culture of control and smpd3-/- mice - as compelling in vitro system - were instrumental in unraveling the molecular mechanism underlying the crucial function of SMPD3 in the GSP. The absence of SMPD3 suppressed ECM protein transport and secretion, disrupted proteostasis, activated UPR and ER stress and finally apoptosis, which is reflected phenotypically in skeletal growth inhibition and joint malformation. Translation of this mechanism into disruption of the GSP in hypothalamic proteo-hormones secreting neurons provides a molecular interpretation of the previously described hypothalamus induced combined pituitary hormone deficiency, underlying the systemic hypoplasia of the smpd3-/- mutant. $^{13}$ 

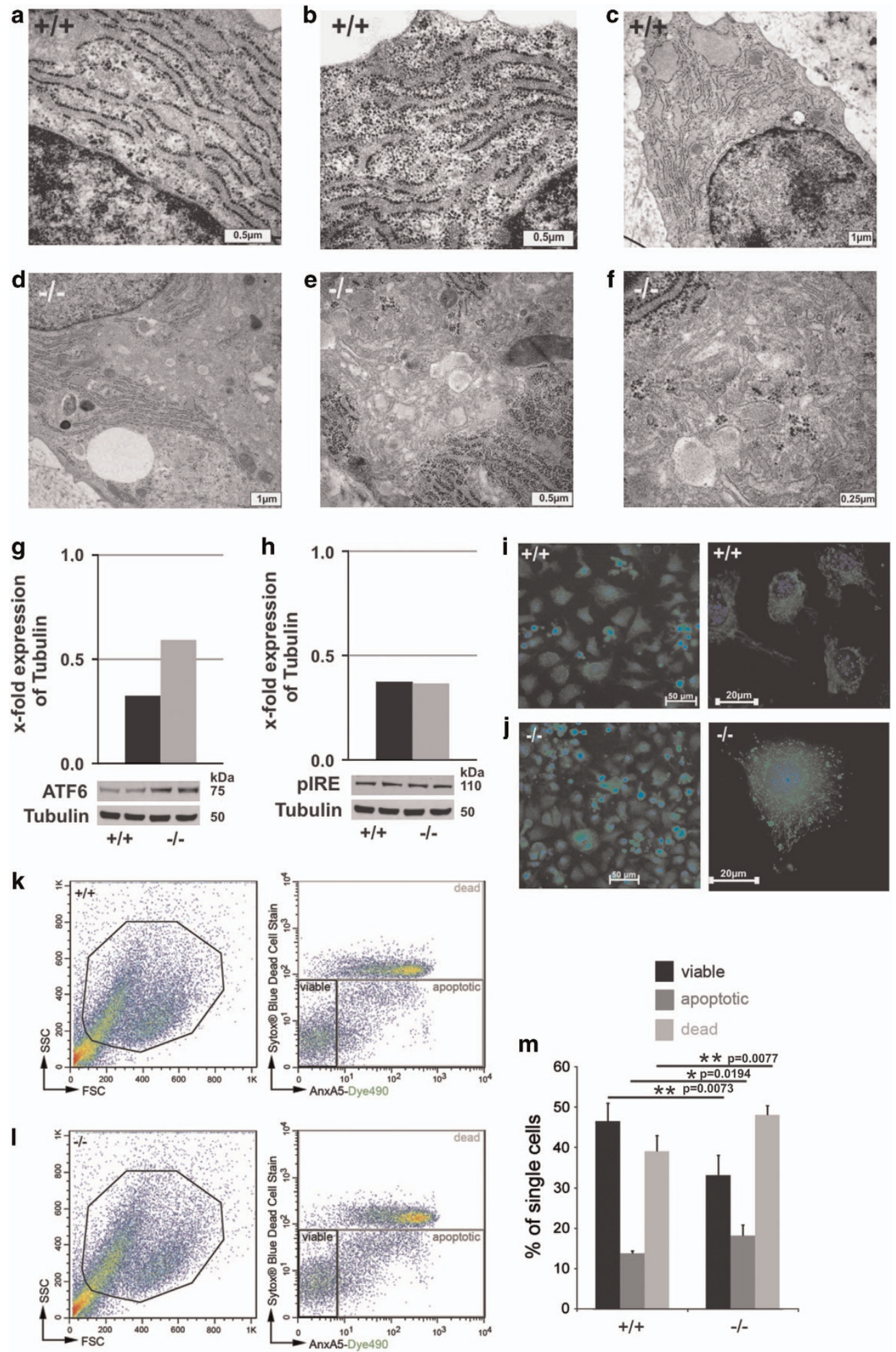

Figure 4 Perturbed proteostasis, UPR, ER stress and apoptosis in primary smpd3 - / - chondrocytes. (a-c) EM displays regular rER in control chondrocytes and (d-f) dilated and giant ER cisternae in smpd3 - / - chondrocytes, $N=3$. ( $\mathbf{g}$ and $\mathbf{~}$ ) WB of control and smpd3 - / - chondrocytes of ATF6 and pIRE, $N=3$. (i and j) Annexin $V$ staining of control and smpd3 - / - chondrocytes. Flow cytometry of single-cell primary chondrocytes from control (upper panel) and smpd3 - / - (lower panel) mice, using AnxA5Dye490 and SYTOX Blue Dead Cell Stain. Representative dot plots are shown. (k) Cell debris were excluded according to size (FSC) and granularity (SSC), followed by gating of single cells (data not shown). (I) AnxA5 $5^{(-)} /$Sytox Blue $e^{(-)}$viable, AnxA5 $5^{(+)} / S y t o x$ Blue ${ }^{(-)}$apoptotic and AnxA5 $5^{(+)} / S y t o x$ Blue ${ }^{(+)}$dead cells were detected. (m) Mean percentage of AnxA5 $5^{(-)} /$Sytox Blue ${ }^{(-)}$viable, AnxA5 $5^{(+)} /$Sytox Blue ${ }^{(-)}$apoptotic and AnxA5 ${ }^{(+)} /$Sytox Blue ${ }^{(+)}$dead cells \pm S.D. is given for control $(+/+)$and smpd3 $-/-$animals, $N=4$. TUNEL assay in sections of radial epiphysis. ( $\mathbf{n}$ and $\mathbf{q})$ negative control, ( $\mathbf{o}$ and $\mathbf{r}$ ) positive control and ( $\mathbf{p}$ and $\mathbf{s}$ ) assay of control and smpd3 $-/-$ chondrocytes, $N=4$. (t and $\mathbf{u})$ Light microscopy of HE-stained paraffin sections $(5 \mu \mathrm{m})$ of decalcified tibial epiphysis of control and smpd3-/ - mice (4mo), $N=4$ 

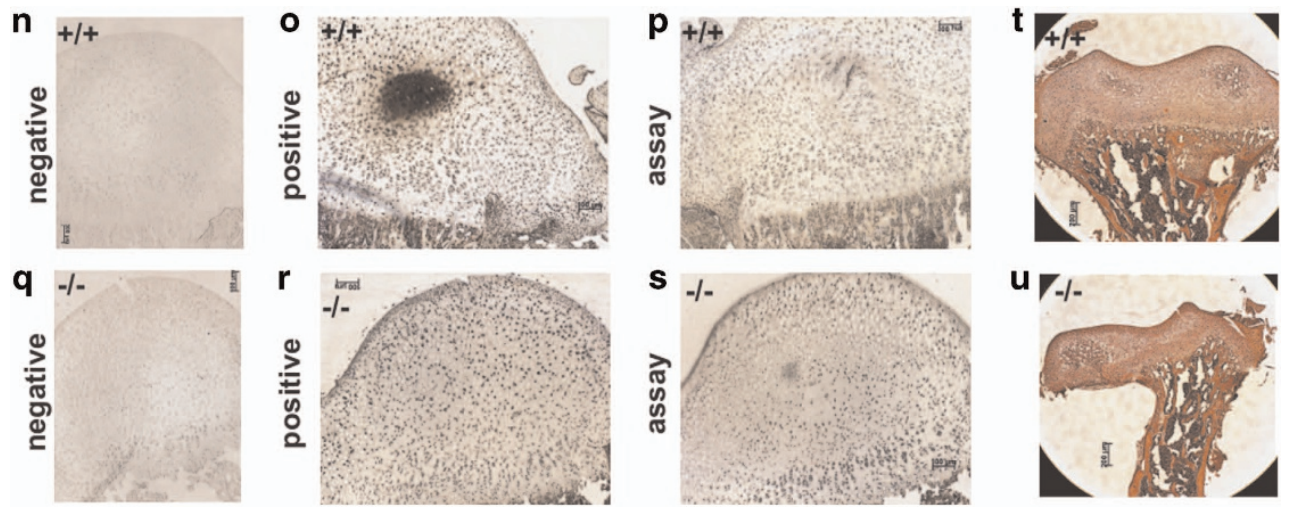

Figure 4 (Continued)

Exploring the function of SMPD3 in the non-canonical GSP, we quantified cytokine secretion in the medium of control and smpd3-/- peritoneal macrophages. The unaltered secretion indicated the restriction of SMPD3 function to the canonical GSP (Figures 10 and $p$ ).

SMPD3 is the dominant among the four mammalian nSMases (SMPD2-5), ${ }^{1-4}$ contributing $>90 \%$ of total cellular nSMase activity in all mouse tissues, followed by SMPD2. ${ }^{13}$ Gene expression, protein expression and nSMase enzyme activity studies on SMPD4 overexpressing HEK293 cells, reported here, preclude SMPD4 as nSMase (Supplementary Figure S1).

Conclusive data on the subcellular topology are a prerequisite for functional studies, as this issue has been controversially discussed and the PLM proposed as the scaffold of SMPD3. ${ }^{18,19}$ The immune-histochemical and biochemical studies, reported here, further suggested the localization and association of SMPD3 and SMS1 in the Golgi complex.

SMPD3 and SMS1 are concentrated in DIMs of the GC of control chondrocytes. In the absence of SMPD3, SMS1 segregates from DIM domains, which disturbed structure of DIMs and deregulate SM synthesis in the GC (Figure 1n). SMS1 protein concentration and enzyme activity were increased in lysates of smpd3-/- chondrocytes (Figures $5 \mathrm{k}$ and I). SM and Cer concentrations were reduced (Figure 2i).

Free DAG species resembled that of the DAG core of PC, the donor substrate in the SMS1 reaction. SMPD3 deficiency caused no SM storage and the phospholipidome in all tissues of smpd3-/- mice remained unimpaired, unlike the fatal lysosomal SM storage in the smpd1 - /- mouse, ${ }^{14,15}$ a mimicry of human Niemann-Pick disease (Figures 2a-h). ${ }^{14,15}$

Disrupted protein transport in GSP of SMPD3-deficient chondrocytes. Chondrocytes are competent secretory cells, actively synthesizing and secreting ECM proteins for epiphyseal cartilage architecture ${ }^{28}$ during the growth phase. The impaired GSP in smpd3-/- chondrocytes causes cytoplasmic accumulation of dominant ECM proteins: fibrillar Col2a, perifibrillar COMP, Matn3, ColVI and CollX (Figures 3a-d). Dysproteostasis activates UPR (Figure 4g), unable to alleviate ER stress, but induces premature apoptosis in smpd3-/- chondrocytes (Figures 4i-u).
EM convincingly unveiled the morphological changes, reflecting these processes: the dense coherent $r E R$ tubular system in control chondrocytes (Figures $4 a-c$ ) is contrasted by inflated cisternae of the rER network, loaded with polymorphic macro-vesicular, fibrillar and granular structures dispersed in the cytoplasm of smpd3-/- chondrocytes (Figures 4d-f). This finally leads to skeletal growth retardation, malformation and chondrodysplasia (Figures $4 \mathrm{t}$ and $\mathrm{u}$ ).

Gene and protein expression of representative ECM proteins Col2a and COMP, Golgi-micro vesicular transport proteins Arf and $\beta$-Cop1 and of growth factor Igf1 and IgfR were inconspicuous (Figures $5 \mathrm{~d}-\mathrm{i}$ ). Expression of growth factors, bmp1, bmp4 and ECM protein comp, respectively, is downregulated (Figure 5c). Secreted BMP1 acts as $\mathrm{Ca}^{2+}$ and $\mathrm{Zn}^{2+}$-dependent metalloproteinase processing procollagen I for ECM organization. SMPD3 is selectively required for the canonical Golgi-mediated secretory pathway. Evidence for unimpaired secretion of non-canonical secretory proteins was provided by multi-cytokine assay, quantifying of 23 cytokines, secreted into the growth-medium of unstimulated and LPS-stimulated peritoneal macrophages of control and smpd3-/- mice, which revealed closely similar concentrations, presented in Figures 10 and $p$.

SMPD3 transiently modifies the Golgi membrane for vesicular transport. The Golgi complex is a budding organelle with domain formation by lateral diffusion. Coatomer complex COPI and II drive budding, fission and fusion in the formation of small vesicular transport carriers. ${ }^{29}$ The function of the SMPD3-SMS1 cycle in the formation of large pleiomorphic carriers for intracellular transport and secretion of ECM proteins in the GSP of chondrocytes, reported here, is a novel facet in studies addressing this enigmatic process.

The central role of SMPD3 and its concerted activity with SMS1 in the SMPD3-SMS1 cycle, which maintains homeostasis of SM metabolism in the GC, is delineated in Figure 6.

Current methodology precludes space- and time-resolved analysis of Golgi membrane domains undergoing remodeling during Golgi vesicular transport. Backed by biochemical, cellular and morphological finding, we conclude that the SMPD3-SMS1 cycle generates a PLC-independent DAG pool in the GC. It is of note that the species of this DAG pool largely reflect those of the major $P C$ species, donor substrates in the 

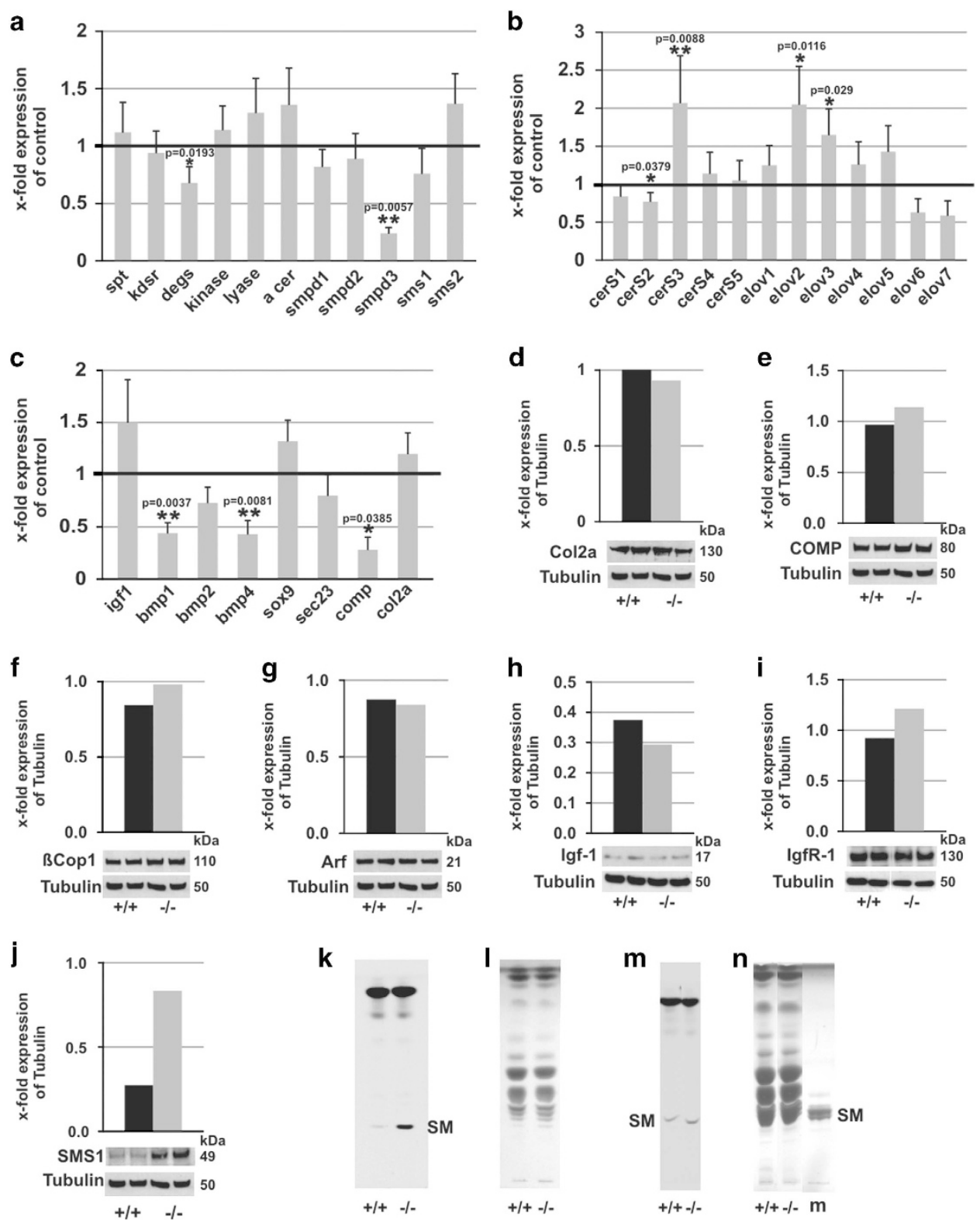

Figure 5 Gene expression profiling of enzymes of SL metabolism, transcription factors, growth factors and ECM proteins in control and smpd3-/ - chondrocytes. Real-time PCR of control and smpd3-/- chondrocytes of (a) spt2 (serine-palmitoyl-CoA transferase), kdsr (3-ketodihydrosphingosine reductase), degs (4t-dihydrosphingosine desaturase), kinase (sphingosine-1-kinase), lyase (sphingosine-1-phosphate lyase), a-cer (acid ceramidase), smpd1, smpd2, smpd3, sms1 and sms2. (b) cerS 1-5 and elov11-7 (fatty acid elongases) and (c) igf1, bmp1, 2, 4, sox9, sec23, comp and col2a. Primers are listed in Supplementary Table S1. Expression was normalized to HGPRT, relative expression calculated using the 2- $\Delta \Delta$ Ct method, \pm S.D., $N=5$. (d-j) Densitometry of WB signals of Col2a, COMP, $\beta$-Cop1, Arf, Igf-1, IgfR-1 and SMS1 in chondrocyte lysates of control (black) and smpd3-/- mice (gray), $N=3$. SMS1 activity in post-mitochondrial fraction of control and smpd3 - / - chondrocytes (k) and kidney (m) using NBDfluorescence $\mathrm{C}_{6}$-Cer (6-((N-(7-nitrobenz-2-oxa-1,3-diazol-4-yl)amino)hexanoyl)sphingosine) as substrate. HPTLC-separation and visualization as inverted images. (I and $\left.\mathbf{n}\right)$ Lipid classes in $\mathrm{k}$ and $\mathrm{m}$ were visualized by charring, $\mathrm{N}=3$

SMS1 catalyzed transfer of the phosphoryl-choline head group to Cer (Figures $2 \mathrm{~d}$ and e), unlike the cellular PI pool of control and smpd3-/- chondrocytes, which contained 18:0/20:4-DAG core as major species (Supplementary Figure S3), ${ }^{30}$ the well-known activator of protein kinases $(\mathrm{PKC})^{31}$ in the reversible recruitment of DAG-responsive proteins with $\mathrm{C} 1$ subdomains to $\mathrm{PLM}{ }^{30-32}$ Potential intricate regulatory functions of Cer and DAG in the Golgi complex await further investigations.
SM/C enriched DIMS of GC membranes are the scaffold of SMPD3 and SMS1 proteins (Figures 1i-k). Sophsticated atomic force microscopy and fluorescence correlation spectroscopy have been applied to phase-separated lipid bilayer model systems, consisting of ternary SM/PC/C domains, embedded in a liquid disordered PC phase, to investigate the effect of Cer on liquid-ordered membrane domains. ${ }^{33-34}$ Treatment with bacterial nSMase released Cer from SM of the SM/C complex and simultaneously displaced cholesterol 


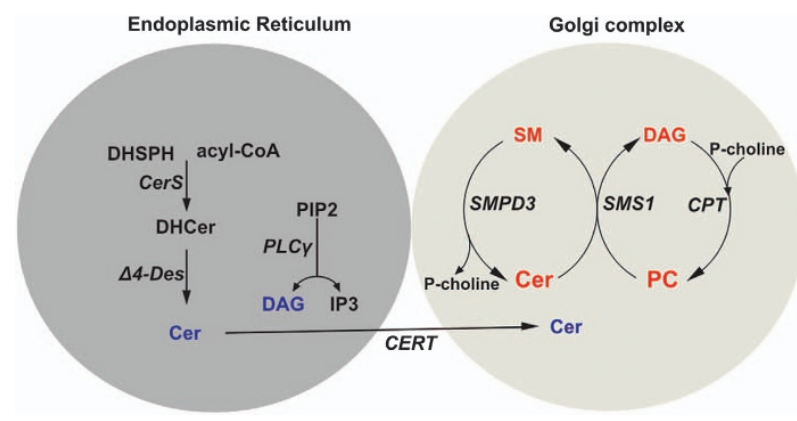

Figure 6 Proposed mechanism of the SMPD3-SMS1 cycle in the Golgi complex. Proposed mechanism of maintenance of homeostasis and regulation of SM, PC, Cerand DAG-pools in the SMPD3-SMS1-cycle of the Golgi complex

stoichiometrically at the rim of ordered nanoscale domain structures. It is tempting to correlate results obtained from these model systems with those of this study on the role of SMPD3 in liquid-ordered DIMS of the Golgi membrane complex.

Complementary and in support of our results are the observations that protein trafficking to the cell surface is retarded in response to downregulation of SMS1, ${ }^{36}$ and the Golgi secretory function inhibited at reduced DAG levels. ${ }^{30}$

SMPD3 induced changes in the homeostasis of SM/PC/ Cer/DAG concentrations in the lipid pool of the $\mathrm{GC}$ are expected to be minor and quantification impeded by insufficient current methodology. Kinetic studies of Cer and DAG release, SM hydrolysis and phosphoryl-choline group transfer from PC in control and SMPD3-deficient GC membrane stacks may give insight into the molecular mechanism underlying the potential role of SMPD3 in the Golgi SMPD3/SMS1 cycle in remodeling the Golgi lipid bilayer membrane for vesicle formation, transport and secretion.

Collectively, these studies suggest a novel mechanism underlying the function of SMPD3 in the GSP and necessitate future experiments addressing the concerted action of SMPD3, SMS1, and of Cer and DAG lipid-driven remodeling of the membrane bilayer in the GSP.

This unprecedented aspect of SMPD3 function necessitates a reconsideration of the current tenet regarding the role of Smases and SM metabolites in intracellular signal transduction pathways. Expansion of this concept to other rapidly growing cells, for example, in tumors, the immune system and inflammation, awaits further studies and may open new perspectives for therapeutic strategies.

\section{Materials and Methods}

Mouse experiments. All experiments were approved by the Institutional Animal Care and Research Advisory Committee of the University of Cologne, Cologne, Germany.

Generation and genetic characterization of smpd3-/- mice (C57B//6 $\times 129$ background) have been described. ${ }^{13}$ They were backcrossed ten times into the C57BL/6 background.

Egfp-N2 fusion constructs. Full-length smpd3cDNA, ${ }^{2}$ inserted into the pcDNA $3.1 \mathrm{myc} / \mathrm{his}$ vector, was amplified with primers NSM2 $5^{\prime}$ Xhol-sense (5'-CTCGAGATGGTTTTGTACACGACCCCCTTTCTT-3') and NSM2 $3^{\prime} \mathrm{Kpnl}$ as (5'-GGTACCACGCCTCCTCTTCCCCTGCAGACACCA-3') and ligated into the Xhol-Kpnl restricted pEGFP-N2 vector.
Full-length smpd4 RNA was amplified by RT-PCR using primers smpd4sXhol 5'-CCCTCGAGGTCTGCTATGGCGTTCCCTCAC-3' and smpd4 as BamH 5'-ATGGCCAGCAGCTCGGATCCGGTGCAGCTT-3'. Smpd4 cDNA was ligated into the PCR2.1 Vector (Invitrogen, Karlsruhe, Germany), isolated as BamHI-Xhol fragment and ligated into the pEGFP-N2 vector.

Transfection. C57BI/6 embryonic fibroblasts (EMFIs) and HEK293 cells were grown in Dulbecco's modified Eagle's medium supplemented with $10 \%$ horse serum, and transfected by electroporation with expression vectors smpd3-EGFP-N2 and smpd4-EGFP-N2.

Real-time PCR. RNA was isolated for RT-PCR from p16 control and smpd3-/ - chondrocytes using Trizol (Invitrogen), reverse-transcribed using a Transcriptase kit (Life Technologies Inc., Darmstadt, Germany). ${ }^{37}$ Quantitative PCR reactions were performed with the ABI Prism $7900 \mathrm{HT}$ using a 96-well format, the Fast SYBR Green Master Mix (Applied Biosystems, Waltham, MA, USA), following the manufacturer's protocol. Data analysis was performed using the 2- $\Delta \Delta \mathrm{Ct}$ method.

Acid and neutral sphingomyelinase assays. Total aSMase- and $\mathrm{Mg}^{2+}$-dependent nSMase activity were determined as described. ${ }^{38}$

SM synthase assay. SMS1 activity in cell lysates was determined following an established procedure. ${ }^{23}$

Cell fractionation. Cell fractionation and isolation of Golgi fractions and Triton $\mathrm{X}$-100 insoluble DIMs were performed by established procedures ${ }^{39-41}$

Cytokine analysis. Cytokines, secreted by peritoneal macrophages via the non-Golgi-secretory pathway, were quantitated in the medium using the BioPlexPro mouse cytokine 23-plex assay (Bio-Rad, Bioplex \#M600009RDPD, Hercules, CA, USA) following the manufacturer's protocol. Macrophages were isolated from adult (4mo) male control and smpd3 - / - mice $72 \mathrm{~h}$ after intraperitoneal injection of $1.5 \mathrm{ml} 4 \%$ thioglycolate, washed with PBS. In all, $1.5 \times 10^{6}$ macrophages unstimulated and stimulated with $100 \mu \mathrm{g} \mathrm{LPS} / \mathrm{ml}$ medium for $48 \mathrm{~h}$ were used. The supernatant was centrifuged for the cytokine assay.

Cell culture. EMFIs (3-6 passage) derived from e14 C57b//6, C57bl/6 $\times 129$ smpd3 - / - (ref.13) and HEK293 cells were grown in Dulbecco's modified Eagle's medium (Sigma, Taufkirchen, Germany), supplemented with $10 \%$ fetal calf serum (Life Technologies Inc.), $2 \mathrm{mmol}$ glutamine, $100 \mathrm{units} / \mathrm{ml}$ penicillin and $100 \mu \mathrm{g} / \mathrm{ml}$ streptomycin in a humidified incubator at $37^{\circ} \mathrm{C}$ in a $5 \% \mathrm{CO}_{2}$ atmosphere. Primary chondrocyte from p16 control and smpd3 $-/$ - of femur epiphyseal cartilage and rib cages were cultured following established procedures. ${ }^{22}$

Lipidomic analysis. Isolation, separation, identification and quantification of phospho classes and SL classes were analyzed by MS/MS using an Applied Biosystems QTrap analyzer (Darmstadt, Germany). ${ }^{41}$ In brief, total lipids were isolated following the method of Bligh and Dyer. ${ }^{42}$ Phospho and SLs were separated by HPTLC in solvent system chloroform/ethanol/triethylamine/water 60/70/70/14 (v/ $\mathrm{v} / \mathrm{v} / \mathrm{v}$ ). Bands were identified by charring with $10 \% \mathrm{CuSO}_{4} .5 \mathrm{H}_{2} \mathrm{O}, 8 \% \mathrm{H}_{3} \mathrm{PO}_{4}$ at $180^{\circ} \mathrm{C}$ for 5 min and quantified using the CAMAG Scanner and software (CAMAG, Muttenz, Switzerland). ${ }^{43}$

Lipid bands were visualized with primuline for analysis by MS/MS using an Applied Biosystems QTrap analyzer. ${ }^{41}$

Protein analysis by western blotting. Protein aliquots were analyzed by WB, using the following antibodies: anti-SMS1, anti-lgf1R and anti-lgf (Santa Cruz Biotechnology, Heidelberg, Germany), anti-MSH (Dianova, Hamburg, Germany), anti-Col2a (Millipore, Darmstadt, Germany), anti-COMP, anti-ColVI, anti-CollX (kindly provided by M Paulsson, Center for Biochemistry, University of Cologne, Cologne, Germany), anti-Arf and anti-Annexin V-Cy3 Apoptosis Detection Kit (Alexis Biochemicals, San Diego, CA, USA), anti-GHRH (Inserm, Paris, France), anti-pIRE and anti-ATF6 (Abcam, Cambridge, UK), anti-Caveolin (BD Transduction Laboratories, Heidelberg, Germany), anti-K58 and anti- $\beta$-Cop (Sigma, Taufkirchen, Germany), affinity purified anti-SMPD2 and SMPD3 (Ser310 - Ala655) polyclonal antibodies, ${ }^{1}$ mouse anti-matrilin 3 (Matn3) antibodies (kindly provided by R. Wagener, ${ }^{44}$ Center for Biochemistry, Cologne, Germany). Quantification was carried out by densitometry using the IMAGE J2X program (Rawak Software, Informer Tech Inc). 
Immunofluorescence microscopy. Primary chondrocytes of p10 control and homozygous female smpd3-/ - mice were grown to semiconfluency on cover slips or on Aclar membranes, respectively, fixed for processing for light and immunofluorescence microscopy with $4 \%$ paraformaldehyde in PBS and permeabilized with $\mathrm{PBS} / 0.5 \%$ Triton X-100, $4{ }^{\circ} \mathrm{C}$. After blocking with $3 \% \mathrm{BSA}$ PBS, cells were treated with respective antibodies in TBS supplemented with $5 \%$ non-fat dry milk at $4{ }^{\circ} \mathrm{C}$ over-night. After washing with PBS/ $0.5 \%$ Triton X-100, cells were incubated with Cy3-conjugated second IgG antibody (Jackson Immuno Research, Baltimore, PA, USA) for $1 \mathrm{~h}$ at $37^{\circ} \mathrm{C}$, washed with PBS/0.5\% Triton $\mathrm{X}-100$, and analyzed by epifluorescence using a Zeiss Axioplan Imager M1 (Oberkochen, Germany) or Leica confocal microscope (Wetzlar, Germany).

FACS analysis of single-cell chondrocytes. For cell survival experiments, cultured primary chondrocytes were analyzed by flow cytometry as previously described. ${ }^{45,46}$ Briefly, chondrocytes were isolated by collagenase type 2 treatment (Worthington Biochemical Corporation, Lakewood, NJ, USA) and subsequently stained with fluorescently labeled annexin A5 (AnxA5-Dye490) ${ }^{2}$ and Sytox Blue Dead Cell Stain (Thermo Fisher Scientific, Schwerte, Germany) in binding buffer (10 mM HEPES, $140 \mathrm{mM} \mathrm{NaCl}, 2.5 \mathrm{mM} \mathrm{CaCl}_{2}$, pH 7.4) followed by flow cytometry analysis (FACSCantoll, Becton Dickinson, Heidelberg, Germany). Using the FlowJo 7.6 software (LLC Ashland, OR, USA), cell debris were excluded and by comparing the area and width of the FSC and SSC signals only single cells were considered for further analysis. Viable (AnxA5/Sytox Blue ${ }^{-}$), apoptotic (AnxA5 $5^{+} /$Sytox Blue ${ }^{-}$) and dead cells $\left(\mathrm{AnxA5}^{+} / \mathrm{Sytox}\right.$ Blue ${ }^{+}$) were quantified and data represented as mean $\pm \mathrm{S}$. D. of $n=4$ individual mice with three technical replicates each. Statistical analysis was performed with Student's t-test using the unpaired two tails method.

Electron microscopy. Primary chondrocyte were grown on Aclar-membranes (Fa. TED Pella Inc., Redding, CA, USA) for 6 days, washed $3 x$ with PBS, fixed for $1 \mathrm{~h}$ at $4{ }^{\circ} \mathrm{C}$ with $2 \%$ glutaraldehyde, $2 \%$ PFA, $0.2 \%$ picric acid in $0.1 \mathrm{M}$ cacodylate buffer, $\mathrm{pH}$ 7.35. Fixation buffer was removed and cells washed $3 \mathrm{x}$ with $0.1 \mathrm{M}$ cacodylate buffer, $\mathrm{pH} 7.35$ post-fixed in $1 \% \mathrm{OsO}_{4}$ solution for $1 \mathrm{~h}$, and stained in $1 \%$ uranyl acetate for $1 \mathrm{~h}$ at room temperature. After dehydration, specimens were embedded in Araldite (Serva, Heidelberg, Germany). Ultra-thin sections (about $70 \mathrm{~nm}$ ) were stained with uranyl acetate and lead citrate and were examined by EM (Zeiss 902A, Zeiss, Oberkochem, Germany). The semi-thin sections $(1 \mu \mathrm{m})$ were stained with methylene blue for light microscopy.

Statistical analyses. Results are expressed as mean \pm S.D. Statistical significance of differences between two groups was determined by a two-tailed Student's t-test using GraphPad QuickCalcs (La Jolla, CA, USA): t-test calculator. A $P$-value of ${ }^{*} \leqslant 0.05,{ }^{* *} \leqslant 0.01,{ }^{* *} \leqslant 0.001$ was considered significant. Sizes of animal cohorts are listed under respective figures.

\section{Conflict of Interest}

The authors declare no conflict of interest.

Acknowledgements. We thank M Paulsson and B Brachvogel, Center of Biochemistry, University of Cologne, for valuable discussion. We gratefully acknowledge the support of this work by the Center of Molecular Medicine, University of Cologne, CECAD (Cluster of Excellence, Cellular Stress Response in Aging-Related Diseases), University of Cologne and Deutsche Forschungsgemeinschaft (Sto32/38-2), JE KÖLN FORTUNE (136/2013, 120/2014) and FZ SFB829, project B11.

1. Tomiuk S, Hofmann K, Nix M, Zumbansen M, Stoffel W. Cloned mammalian neutral sphingomyelinase: functions in sphingolipid signaling? Proc Natl Acad Sci USA 1998; 95 3638-3643.

2. Hofmann K, Tomiuk S, Wolff G, Stoffel W. Cloning and characterization of the mammalian brain-specific, Mg2+-dependent neutral sphingomyelinase. Proc Natl Acad Sci USA 2000; 97: 5895-5900.

3. Krut O, Wiegmann K, Kashkar H, Yazdanpanah B, Kronke M. Novel tumor necrosis factorresponsive mammalian neutral sphingomyelinase-3 is a $\mathrm{C}$-tail-anchored protein. $\mathrm{J}$ Biol Chem 2006; 281: 13784-13793.

4. Wu BX, Rajagopalan V, Roddy PL, Clarke CJ, Hannun YA. Identification and characterization of murine mitochondria-associated neutral sphingomyelinase (MAnSMase), the mammalian sphingomyelin phosphodiesterase 5. J Biol Chem 2010; 285 : 17993-18002
5. Hannun YA, Obeid LM. Many ceramides. J Biol Chem 2011; 286: 27855-27862.

6. Galadari S, Rahman A, Pallichankandy S, Thayyullathil F. Tumor suppressive functions of ceramide: evidence and mechanisms. Apoptosis 2015; 20: 689-711.

7. Zumbansen $M$, Stoffel W. Tumor necrosis factor alpha activates NF-kappaB in acid sphingomyelinase-deficient mouse embryonic fibroblasts. J Biol Chem 1997; 272: 10904-10909.

8. Watts JD, Gu M, Polverino AJ, Patterson SD, Aebersold R. Fas-induced apoptosis of T cells occurs independently of ceramide generation. Proc Natl Acad Sci USA 1997; 94: 7292-7296.

9. Hofmann K, Dixit VM. Ceramide in apoptosis-does it really matter? Trends Biochem Sci 1998; 23: 374-377.

10. Nix M, Stoffel W. Perturbation of membrane microdomains reduces mitogenic signaling and increases susceptibility to apoptosis after T cell receptor stimulation. Cell Death Differ 2000; 7: 413-424.

11. Zumbansen $M$, Stoffel $W$. Neutral sphingomyelinase 1 deficiency in the mouse causes no lipid storage disease. Mol Cell Biol 2002; 22: 3633-3638.

12. van Blitterswijk WJ, van der Luit AH, Veldman RJ, Verheij M, Borst J. Ceramide: second messenger or modulator of membrane structure and dynamics? Biochem $J$ 2003; 369: 199-211.

13. Stoffel W, Jenke B, Block B, Zumbansen M, Koebke J. Neutral sphingomyelinase 2 (smpd3) in the control of postnatal growth and development. Proc Natl Acad Sci USA 2005; 102: 4554-4559.

14. Otterbach B, Stoffel W. Acid sphingomyelinase-deficient mice mimic the neurovisceral form of human lysosomal storage disease (Niemann-Pick disease). Cell 1995; 81: 1053-1061.

15. Horinouchi K, Erlich S, Perl DP, Ferlinz K, Bisgaier CL, Sandhoff $\mathrm{K}$ et al. Acid sphingomyelinase deficient mice: a model of types $\mathrm{A}$ and $\mathrm{B}$ Niemann- Pick disease. Nat Genet 1995; 10: 288-293.

16. Stoffel W, Jenke B, Holz B, Binczek E, Günter RH, Knifka J et al. Neutral sphingomyelinase (SMPD3) deficiency causes a novel form of chondrodysplasia and dwarfism that is rescued by Col2A1-driven smpd3 transgene expression. Am J Pathol 2007; 171: 153-161.

17. Genotype-Tissue Expression (GTEx) Project. www.GTExportal.org.

18. Milhas D, Clarke CJ, Hannun YA. Sphingomyelin metabolism at the plasma membrane: implications for bioactive sphingolipids. FEBS Lett 2010; 584: 1887-1894.

19. Tani M, Hannun YA. Analysis of membrane topology of neutral sphingomyelinase 2. FEBS Lett 2007; 581: 1323-1328.

20. Efstratiadis A. Genetics of mouse growth. Int J Dev Biol 1998; 42: 955-976.

21. Posey KL, Coustry F, Veerisetty AC, Liu P, Alcorn JL, Hecht JT. Chondrocyte-specific pathology during skeletal growth and therapeutics in a murine model of pseudoachondroplasia. J Bone Miner Res 2014; 29: 1258-1268.

22. Thirion S, Berenbaum F. Culture and phenotyping of chondrocytes in primary culture. Methods Mol Med 2004; 100: 1-14

23. Huitema K, van den Dikkenberg J, Brouwers JF, Holthuis JC. Identification of a family of animal sphingomyelin synthases. EMBO J 2004; 23: 33-44.

24. Oslowski CM, Urano F. The binary switch that controls the life and death decisions of ER stressed beta cells. Curr Opin Cell Biol 2011; 23: 207-215.

25. Oslowski CM, Urano F. Measuring ER stress and the unfolded protein response using mammalian tissue culture system. Methods Enzymol 2011; 490: 71-92.

26. Riggs AC, Bernal-Mizrachi E, Ohsugi M, Wasson J, Fatrai S, Welling $C$ et al. Mice conditionally lacking the Wolfram gene in pancreatic islet beta cells exhibit diabetes as a result of enhanced endoplasmic reticulum stress and apoptosis. Diabetologia 2005; 48: 2313-2321.

27. Verhoven B, Schlegel RA, Williamson P. Mechanisms of phosphatidylserine exposure, a phagocyte recognition signal, on apoptotic T lymphocytes. J Exp Med 1995; 182: 1597-1601.

28. Zaucke F, Grassel S. Genetic mouse models for the functional analysis of the perifibrillar components collagen IX, COMP and matrilin-3: Implications for growth cartilage differentiation and endochondral ossification. Histol Histopathol 2009; 24 : 1067-1079.

29. Rothman JE, Wieland FT. Protein sorting by transport vesicles. Science 1996; 272: 227-234.

30. Baron CL, Malhotra V. Role of diacylglycerol in PKD recruitment to the TGN and protein transport to the plasma membrane. Science 2002; 295: 325-328.

31. Kishimoto A, Takai Y, Mori T, Kikkawa U, Nishizuka Y. Activation of calcium and phospholipid-dependent protein kinase by diacylglycerol, its possible relation to phosphatidylinositol turnover. J Biol Chem 1980; 255: 2273-2276.

32. Nishizuka Y. Turnover of inositol phospholipids and signal transduction. Science 1984; 225 : 1365-1370.

33. Wang H, Yang C, Leskow FC, Sun J, Canagarajah B, Hurley JH et al. Phospholipase Cgamma/diacylglycerol-dependent activation of beta2-chimaerin restricts EGF-induced Rac signaling. EMBO J 2006; 25: 2062-2074.

34. Chiantia S, Kahya N, Ries J, Schwille P. Effects of ceramide on liquid-ordered domains investigated by simultaneous AFM and FCS. Biophys J 2006; 90: 4500-4508.

35. Ira, Johnston LJ. Sphingomyelinase generation of ceramide promotes clustering of nanoscale domains in supported bilayer membranes. Biochim Biophys Acta 2008; 1778 : 185-197.

36. Subathra M, Qureshi A, Luberto C. Sphingomyelin synthases regulate protein trafficking and secretion. PLoS One 2011; 6: e23644. 
37. Stoffel W, Holz B, Jenke B, Binczek E, Günter RH, Kiss C et al. Delta6-desaturase (FADS2 deficiency unveils the role of omega3- and omega6-polyunsaturated fatty acids. EMBO $J$ 2008; 27: 2281-2292.

38. Tomiuk S, Zumbansen M, Stoffel W. Characterization and subcellular localization of murine and human magnesium-dependent neutral sphingomyelinase. J Biol Chem 2000; 275: 5710-5717.

39. Graham JM. Isolation of Golgi membranes from tissues and cells by differential and density gradient centrifugation. Curr Protoc Cell Biol 2001; Chapter 3(Unit 3): 9.

40. Simons K, Toomre D. Lipid rafts and signal transduction. Nat Rev Mol Cell Biol 2000; 1: 31-39.

41. Melkonian KA, Ostermeyer AG, Chen JZ, Roth MG, Brown DA. Role of lipid modifications in targeting proteins to detergent-resistant membrane rafts. Many raft proteins are acylated, while few are prenylated. J Biol Chem 1999; 274: 3910-3917.

42. Stoffel W, Hammels I, Jenke B, Binczek E, Schmidt-Soltau I, Brodesser S et al. Obesity resistance and deregulation of lipogenesis in Delta6-fatty acid desaturase (FADS2) deficiency. EMBO Rep 2014; 15: 110-120.

43. Bligh EG, Dyer WJ. A rapid method of total lipid extraction and purification. Can J Biochem Physiol 1959; 37: 911-917.

44. Wagener R, Kobbe B, Paulsson M. Primary structure of matrilin-3, a new member of a family of extracellular matrix proteins related to cartilage matrix protein (matrilin-1) and von Willebrand factor. FEBS Lett 1997; 413: 129-134.
45. Etich J, Holzer T, Pitzler L, Bluhm B, Brachvogel B. MiR-26a modulates extracellular matrix homeostasis in cartilage. Matrix Biol 2015; 43: 27-34.

46. Rosenbaum S, Kreft S, Etich J, Frie C, Stermann J, Grskovic I et al. Identification of novel binding partners (annexins) for the cell death signal phosphatidylserine and definition of their recognition motif. J Biol Chem 2011; 286: 5708-5716.

(c) Cell Death and Disease is an open-access journal published by Nature Publishing Group. This work is

licensed under a Creative Commons Attribution 4.0 International License. The images or other third party material in this article are included in the article's Creative Commons license, unless indicated otherwise in the credit line; if the material is not included under the Creative Commons license, users will need to obtain permission from the license holder to reproduce the material. To view a copy of this license, visit http://creativecommons.org/licenses/by/4.0/

(C) The Author(s) 2016

Supplementary Information accompanies this paper on Cell Death and Disease website (http://www.nature.com/cddis) 